\title{
Avifauna del bosque mesófilo de montaña del noreste de Hidalgo, México
}

\author{
Avifauna of the tropical montane cloud forest of northeastern Hidalgo, Mexico
}

\author{
Miguel Angel Martínez-Morales \\ Centro de Investigaciones Biológicas, Universidad Autónoma del Estado de Hidalgo \\ Apartado postal 69, Plaza Juárez, 42001, Pachuca, Hidalgo, México \\ Correspondencia: migmarti@uaeh.edu.mx
}

\begin{abstract}
Resumen. Este trabajo presenta los resultados de un inventario avifaunístico realizado de 1997 a 1999 en fragmentos de bosque mesófilo de montaña del noreste de Hidalgo, México. Se registraron 41 familias y 181 especies de aves mediante observaciones visuales y auditivas en 2057 puntos de conteo, lo que representó el 98\% de las especies esperadas en el área de estudio, para el período y método de muestreo utilizado. Se detectaron 16 especies restringidas al bosque mesófilo, 11 endémicas de México y 3 de distribución restringida. Adicionalmente, con base en la legislación mexicana vigente, 28 de las especies registradas están incluidas dentro de alguna categoría de riesgo de conservación. Esta comunidad de aves estuvo dominada por especies de aves pequeñas, raras (poco abundantes) y residentes. Es probable que las más vulnerables de sufrir extinciones locales sean las especies raras y restringidas al bosque mesófilo, donde están incluidas las 3 especies de distribución restringida (Dendrortyx barbatus, Glaucidium sanchezi y Cyanolyca nana). La avifauna del bosque mesófilo de esta región incluye al $40 \%$ de la avifauna estatal, lo que destaca la relevancia de este tipo de vegetación y una urgente necesidad de establecer estrategias de manejo para su conservación.
\end{abstract}

Palabras clave: aves, población, comunidad, inventario, abundancia, rareza, conservación, bosque de niebla.

\begin{abstract}
This study shows the results of bird census carried out from 1997 to 1999 in cloud forest fragments of northeastern Hidalgo, Mexico. Forty-one bird families and 181 species were recorded through visual and acoustic detections in 2057 point counts. This represents $98 \%$ of the expected species richness for the sampling period and method used. Sixteen species restricted to the cloud forest were detected, 11 Mexican endemic species, and 3 restricted-range species were recorded. Additionally, 28 species are included within some category of conservation concern according to the present Mexican legislation. This bird community was dominated by small, rare (low in abundance), and resident bird species. It is likely that the most vulnerable species to local extinction were those rare species restricted to the cloud forest, including the 3 restricted-range species (Dendrortyx barbatus, Glaucidium sanchezi, and Cyanolyca nana). The avifauna of the cloud forest of this region includes $40 \%$ of the avifauna of the state of Hidalgo, stressing the relevance of this vegetation type and the urgent need for implementing management strategies for conservation.
\end{abstract}

Key words: birds, population, community, census, abundance, rarity, conservation, cloud forest.

\section{Introducción}

De los ecosistemas presentes en México, el bosque mesófilo de montaña contiene una de las avifaunas más ricas por su número total de especies, por la cantidad de endemismos que alberga y porque en él se encuentra una de las proporciones más altas de especies restringidas ecológicamente (Escalante-Pliego et al., 1998). El conocimiento actual de la avifauna del bosque mesófilo en México se ha originado a partir de colectas y censos

Recibido: 17 enero 2006; aceptado: 29 agosto 2006 de aves en varias localidades y tiempos (Chapman, 1898; Sutton y Burleigh, 1940; Wetmore, 1943; Lowery y Newman, 1949; Bjelland y Ray, 1977; Navarro et al., 1991, 2004; Howell y Webb, 1992; Escalona et al., 1995; Gram y Faaborg, 1997; Rojas-Soto et al., 2002; para el este de México). Este conocimiento de la avifauna, aunque parcial, ha constituido la información base para estudios posteriores que han analizado los patrones generales de distribución espacial y temporal de las especies de aves y su endemismo en el bosque mesófilo (Martin, 1955; Navarro, 1992; Navarro et al., 2004). Asimismo, con el fin de definir unidades biogeográficas discretas para este tipo 
de bosque, algunos estudios se han enfocado en la búsqueda de afinidades avifaunísticas entre diferentes regiones de bosque mesófilo o intervalos latitudinales dentro de una misma región (Escalona et al., 1995; HernándezBaños et al., 1995; Navarro et al., 2004). El análisis de la distribución de estas unidades biogeográficas dentro del esquema de las áreas naturales protegidas en México ha identificado los bosques mesófilos del este de México (en los estados de Tamaulipas, San Luis Potosí, Veracruz, Hidalgo, Querétaro, Puebla y norte de Oaxaca) como un área biogeográfica discreta que no ha sido adecuadamente protegida (Challenger, 1998; Martínez-Morales, 2001; Navarro et al., 2004). En años recientes, algunos otros estudios se han enfocado a entender la relación que existe entre los patrones paisajísticos y la diversidad de aves en el bosque mesófilo, con el fin de orientar las estrategias de manejo para la conservación (Martínez-Morales, 2005a, 2005b). Por otra parte, para subsanar la parcialidad en el conocimiento que se tiene de la distribución espacial de las especies de aves, se está trabajando en la estimación de dicha distribución a partir del empleo de modelos predictivos de distribución de especies (datos no publicados).

Las conclusiones a las que llegan los estudios que parten de la información generada de inventarios dependen en buena medida de la calidad de dicha información. En este contexto, el propósito de este trabajo es describir de manera detallada la composición y estructura de la avifauna del bosque mesófilo de montaña del noreste de Hidalgo, como una forma de incrementar el conocimiento que se tiene de la avifauna de este importante ecosistema y como información base para estudios posteriores.

\section{Material y métodos}

Área de estudio. En Hidalgo, el bosque mesófilo se distribuye en la vertiente del Golfo de México de la Sierra Madre Oriental, normalmente a altitudes entre los 1000 y $2000 \mathrm{~m}$, aunque su distribución altitudinal va desde los 750 a 2400 m (Luna-Vega et al., 2000). Si la humedad no es suficientemente alta para el desarrollo del bosque mesófilo, pueden existir bosques de pino-encino o encino dentro de esta banda altitudinal. Debajo de dicha banda, la vegetación tropical reemplaza al bosque mesófilo y por encima de esa banda, los bosques de pino y pino-encino son los tipos de vegetación dominantes.

El bosque mesófilo en Hidalgo es denso y puede presentar 2 o 3 estratos arbóreos. El dosel incluye especies arbóreas perennifolias y caducifolias, con una altura promedio de 10 a $20 \mathrm{~m}$, o más, y un diámetro de 30 a $50 \mathrm{~cm}$. Las especies arbóreas de dosel más importantes pertenecen a los géneros Liquidambar, Quercus, Magnolia,
Pinus, Podocarpus, Clethra, Meliosma, Carpinus, Nyssa, Ostrya, Alnus, Crataegus, Symplocos, Prunus y Phoebe.

En la mayoría de los casos, los bosques prácticamente puros de Liquidambar se consideran como estadios sucesionales hacia el reestablecimiento de bosques mixtos de Liquidambar y otras especies arbóreas. También existen bosques puros de Fagus mexicana que se restringen a altitudes entre 1800 y 1900 m (Miranda y Sharp, 1950; Rzedowski, 1981; Luna-Vega et al., 1994; WilliamsLinera et al., 2003).

Con base en el Inventario Forestal Nacional 2000 (Velázquez et al., 2002), en Hidalgo existe una superficie de aproximadamente 57000 ha de bosque mesófilo y una superficie igual de bosque mesófilo con vegetación secundaria arbustiva y herbácea. La ganadería extensiva ha producido los cambios más importantes en la cobertura del suelo de esta región, debido a la remoción de la vegetación nativa para cultivar o inducir pastizales.

En este estudio, los muestreos fueron realizados en varios fragmentos de bosque mesófilo localizados en el noreste del estado, incluyendo los municipios de Tlanchinol, Calnali, Lolotla, Molango de Escamilla, Xochicoatlán, Tianguistengo y Zacualtipán de Ángeles (Fig. 1). Todos los muestreos se restringieron a una banda altitudinal entre los 1300 y $1900 \mathrm{~m}$, ya que dentro de estos límites se encuentra el bosque mesófilo típico en la región. Las características específicas de cada fragmento estudiado ya se han presentado en otras publicaciones (Martínez-Morales, 2001, 2004, 2005a). El área de estudio se encuentra dentro del área de endemismo de aves denominada Sur de la Sierra Madre Oriental, a la cual se le considera en un nivel crítico de prioridad para el desarrollo de acciones de conservación para las aves (Stattersfield et al., 1998). El Área de Importancia para la Conservación de las aves en México (AICA) Tlanchinol, catalogada dentro de la categoría G-1 por contener especies de aves amenazadas globalmente, está incluida dentro del área de estudio (Arizmendi y Márquez-Valdelamar, 2000). Asimismo, forma parte de la Región Terrestre Prioritaria 102 "Bosques Mesófilos de la Sierra Madre Oriental" (Arriaga et al., 2000).

Trabajo de campo. Excepto por los períodos de octubre a diciembre de cada año, durante el estudio se invirtieron 2 semanas mensuales de trabajo en campo, de junio de 1997 a agosto de 1999. Se utilizaron conteos por punto para estimar la riqueza y abundancia de las especies de aves. Al final del estudio se acumuló un total de 2057 puntos. Los períodos de registro fueron realizados durante 3 horas en la mañana y en la tarde, después y antes del período crepuscular, respectivamente; todos los sitios de muestreo fueron censados en ambos períodos. Los conteos en cada punto tuvieron una duración de 10 minutos, estableciendo 

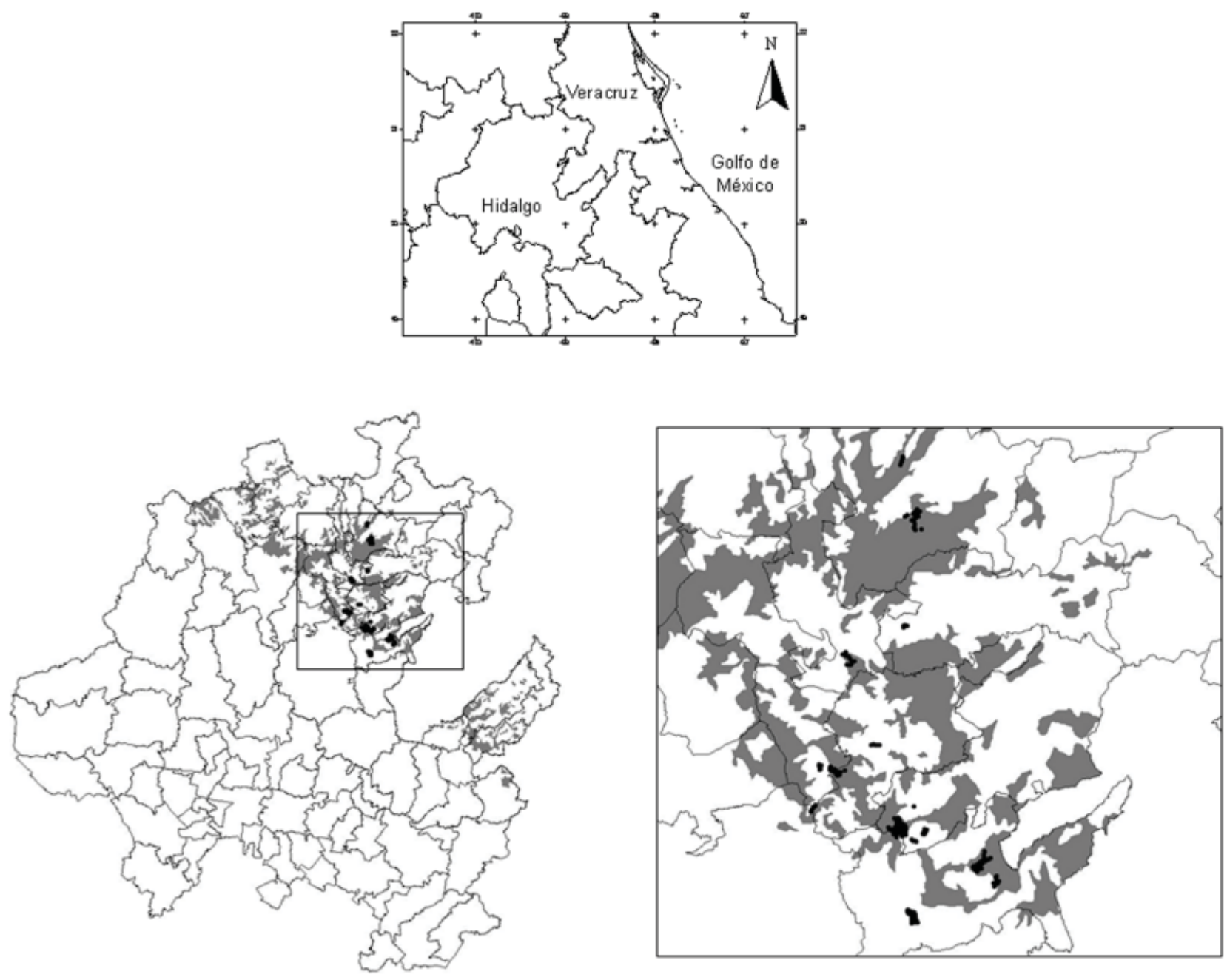

Figura 1. Puntos de registro de especies de aves en fragmentos de bosque mesófilo de montaña del noreste de Hidalgo, México. La superficie de bosque mesófilo se representa en color gris, con base en el Inventario Forestal Nacional 2000 (Velázquez et al., 2002). En los recuadros del estado de Hidalgo se presenta la división política municipal.

una distancia mínima de aproximadamente $200 \mathrm{~m}$ entre puntos adyacentes para asegurar su independencia (Ralph et al., 1995). La ubicación de los puntos se determinó en campo mediante el empleo de un receptor GPS y un altímetro. Para las observaciones se utilizaron binoculares $8 \times 32$, y, cuando fue posible, la distancia de las observaciones se midió con un telémetro óptico. Todos los conteos se realizaron en condiciones ambientales de buena detección visual y auditiva, es decir, sin viento excesivo, lluvia o neblina.

Información de las especies de aves. La nomenclatura y secuencia taxonómica de las especies de aves registradas en este trabajo está basada en $\operatorname{AOU}(1998,2000)$ y Banks et al. (2002, 2003, 2004, 2005). La masa corporal de las especies se obtuvo de Dunning (1993) y de la información de especímenes de la Colección Nacional de Aves del Instituto de Biología, UNAM. El gremio trófico-conductual de cada especie de ave se determinó con base en la información disponible sobre preferencias alimentarias (Howell y Webb, 1995) y las categorías que establecen Terborgh et al. (1990), Kattan et al. (1994) y Nocedal (1994). La definición de la estacionalidad de las especies registradas se basó en las observaciones en campo y en la información bibliográfica disponible (Howell y Webb, 1995). De esta manera, se definieron las siguientes categorías:

- Residente (R). Especie que se reproduce y permanece en el área todo el año.

- Visitante de verano (V). Especie que utiliza el área sólo durante la temporada reproductiva (de febrero a agosto). 
- Residente/visitante de invierno (RI). Especie con poblaciones residentes y poblaciones migratorias neotropicales que utilizan el área como sitio de invernación.

- Visitante de invierno/migratoria de paso (IP). Especie migratoria neotropical con poblaciones que usan el área como sitio de invernación y poblaciones que la usan como zona de paso durante la migración (de agosto a mayo).

- Migratoria de paso (P). Especie migratoria neotropical que usa el área como zona de paso durante la migración (de abril a mayo y de agosto a octubre).

Análisis de datos. Con el fin de evaluar la suficiencia en el esfuerzo de muestreo para detectar la mayoría de las especies presentes, se utilizó una función exponencial de acumulación de especies (Soberón y Llorente, 1993). Esta función supone que la probabilidad de añadir una nueva especie a la lista decrece de manera lineal con el tamaño de la lista, llegando finalmente a cero. Este supuesto es válido en particular cuando se muestrea un área relativamente pequeña o un taxón bien conocido, o ambas situaciones. La función es la siguiente:

$$
S(t)=a / b\left(1-e^{b t}\right)
$$

donde $S(t)$ representa el número esperado de especies después de $t$ puntos de conteo. El parámetro $a$ es la tasa de incremento de especies en la lista al comienzo del estudio y la asíntota está dada por $a / b$. El parámetro $a$ tiene unidades de especies/puntos de conteo y $b$ de puntos de conteo $^{-1}$. Los parámetros $a$ y $b$ se estimaron mediante regresiones no lineales y la función de acumulación de especies se ajustó mediante el método Levenberg-Marquardt (Bates y Watts, 1988).

La abundancia relativa de las especies se consideró como el número medio de individuos detectados en 100 puntos de conteo:

Abundancia relativa $=$ (individuos detectados $/$ número total de puntos de conteo) x 100

Para estimar la abundancia relativa se tomó en cuenta la estacionalidad registrada y observada de cada especie (por ejemplo, especies migratorias) al momento de calcular el número total de puntos de conteo. La estimación de la abundancia relativa de las especies está sesgada por la probabilidad específica de detección, por lo que las comparaciones de estos valores entre especies no son del todo adecuadas. Sin embargo, en términos generales, estas estimaciones son un referente para conocer qué tan abundante es una especie.

La densidad de cada especie fue calculada con base en la teoría del muestreo de distancias (Buckland et al., 1993, 2004). Los datos de las distancias de observación obtenidos en campo para cada especie se emplearon para calcular su densidad mediante el programa Distance 5.0 (Tomas et al., 2005). Los datos fueron analizados como observaciones individuales o grupales (en el caso de parvadas), dependiendo de la especie. Aunque se estimó la densidad de todas las especies para las que existían datos de distancia, sólo se registran las de aquellas para las cuales se obtuvieron estimaciones estadísticamente robustas.

\section{Resultados}

Fueron registradas 41 familias y 181 especies de aves en el área de estudio (Cuadro 1). Las especies de los géneros Cypseloides y Empidonax (excepto E. occidentalis y E. fulvifrons) no fueron tratadas a nivel de especie por la dificultad en su identificación mediante observaciones visuales. Con base en la curva de acumulación de especies (Fig. 2), el número detectado representa el $98 \%$ de la avifauna esperada en el área de estudio (184 especies), para el período y los métodos de muestreo empleados. Esto sugiere que el esfuerzo de muestreo invertido fue adecuado para prácticamente detectar todas las especies que se esperaban. Es probable que algunas no hayan sido registradas debido a que sólo se llevaron a cabo detecciones visuales y auditivas, a que todos los muestreos fueron diurnos, y a que el período de estudio sólo comprendió los meses de enero a septiembre. Por lo tanto, las especies difíciles de identificar de manera visual o auditiva, las crípticas, las nocturnas y las migratorias latitudinales pueden estar subrepresentadas en alguna medida.

Como resultado de este estudio, se obtuvieron nuevos registros y ampliaciones en el área de distribución de 6 especies: Harpagus bidentatus, Glaucidium sanchezi, Attila spadiceus, Cyanolyca nana, Oreoscoptes montanus y Peucedramus taeniatus (Martínez-Morales, 2004).

La masa corporal de esta avifauna varió de 2 g (Atthis heloisa) a más de $2 \mathrm{~kg}$ (Penelope purpurascens); sin embargo, la mayor parte de las especies detectadas tuvieron una masa corporal menor a los $80 \mathrm{~g}$, es decir, que esta comunidad está dominada por especies de aves pequeñas (Fig. 3).

En esta comunidad se definieron 34 gremios tróficoconductuales (Cuadro 2). El gremio con el mayor número de especies fue el de los insectívoros arborícolas de follaje con 26 especies de las familias Paridae, Aegithalidae, Troglodytidae, Regulidae, Sylviidae, Peucedramidae y Parulidae. Otros gremios importantes fueron los de insectívoros-frugívoros arborícolas de follaje con 14 especies de los géneros Vireo, Vireolanius, Cyclarhis, Sialia, Myadestes, Catharus, Turdus y Dumetella; de nectarívoros-insectívoros, que incluyó todos los colibríes, 


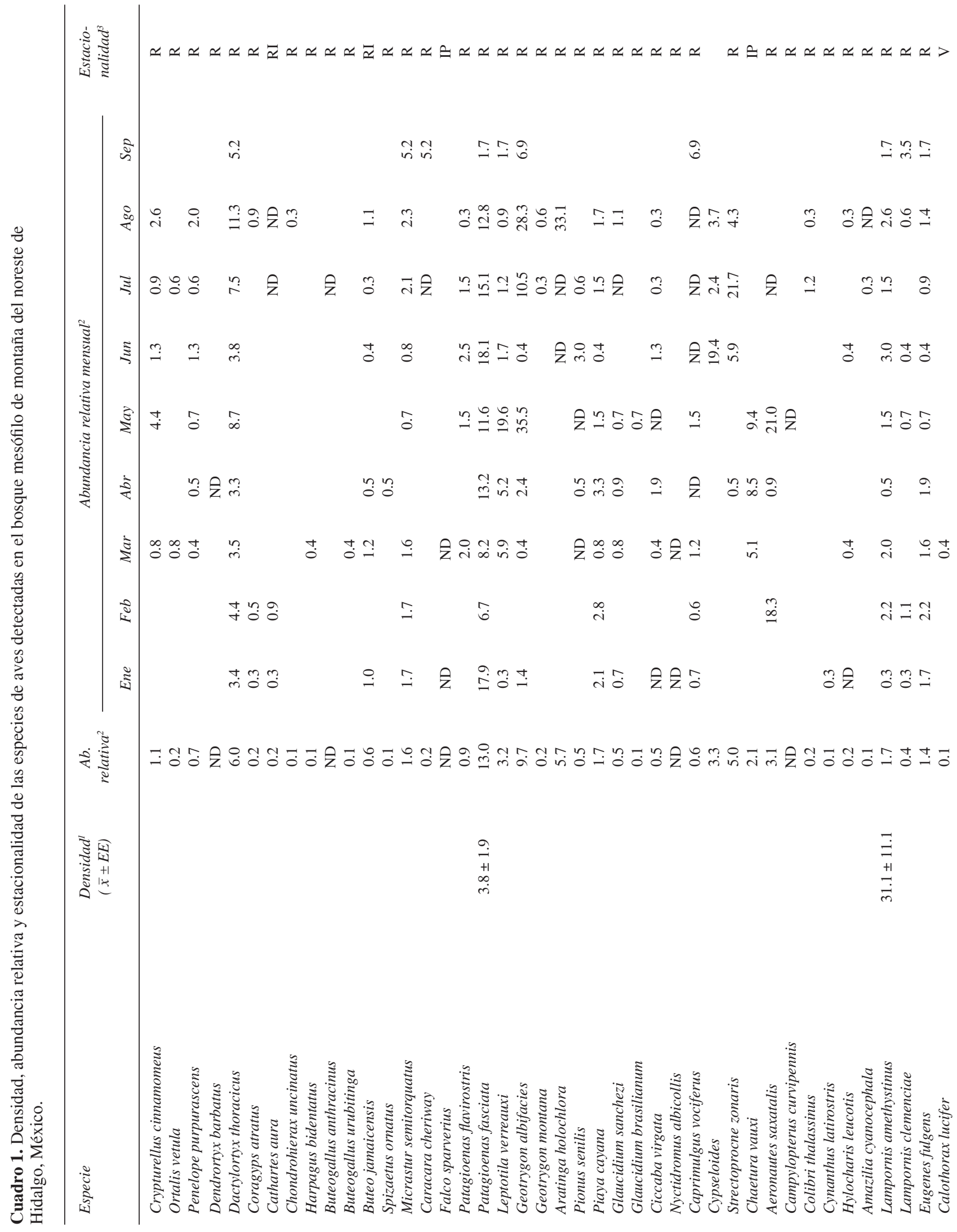




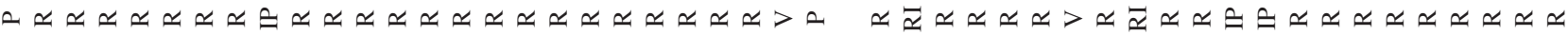

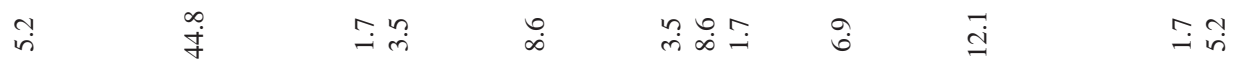

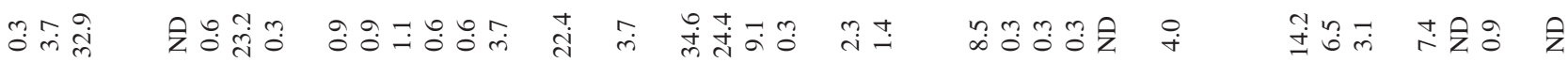
ํํㄱ :

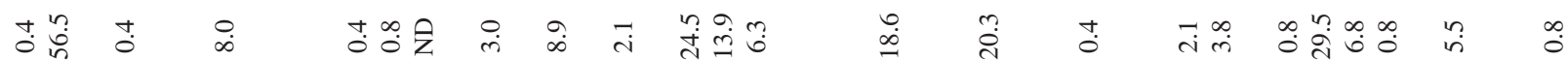

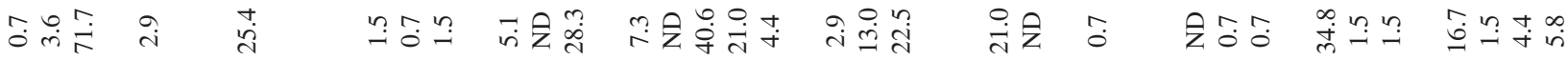

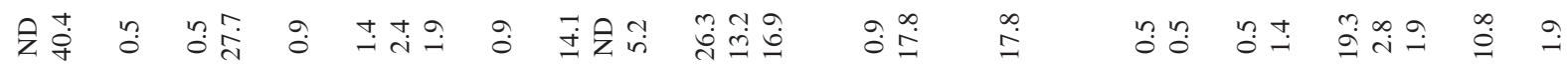

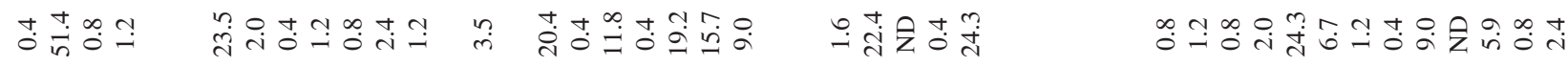
芹递

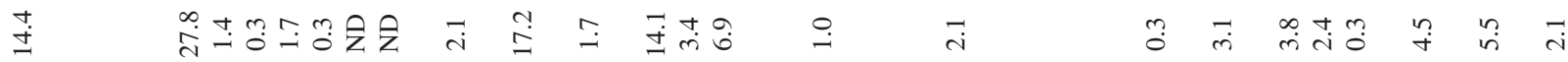

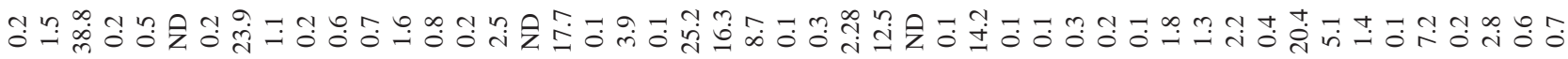

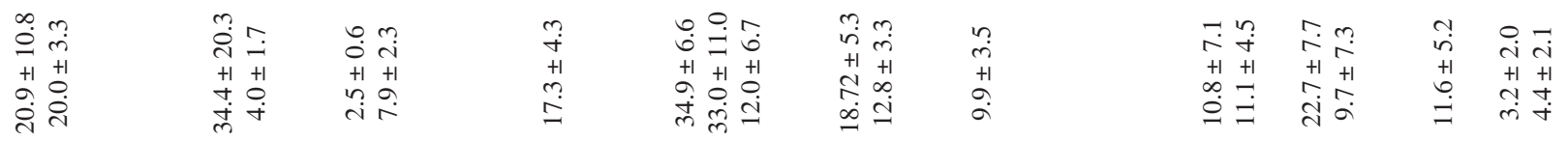

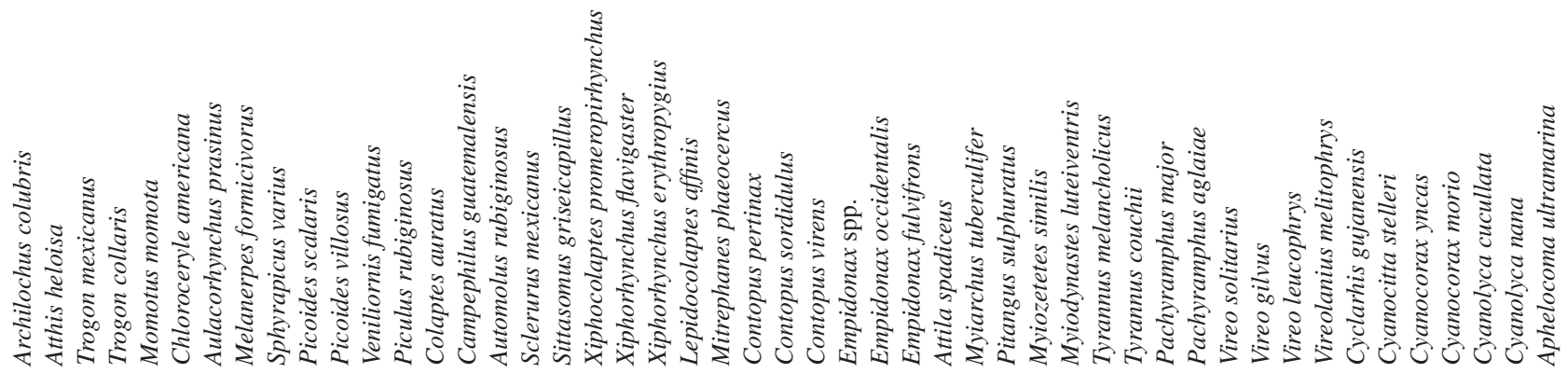




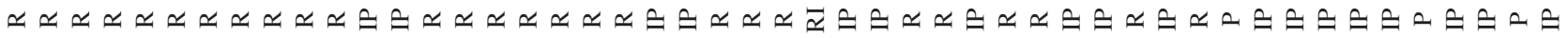

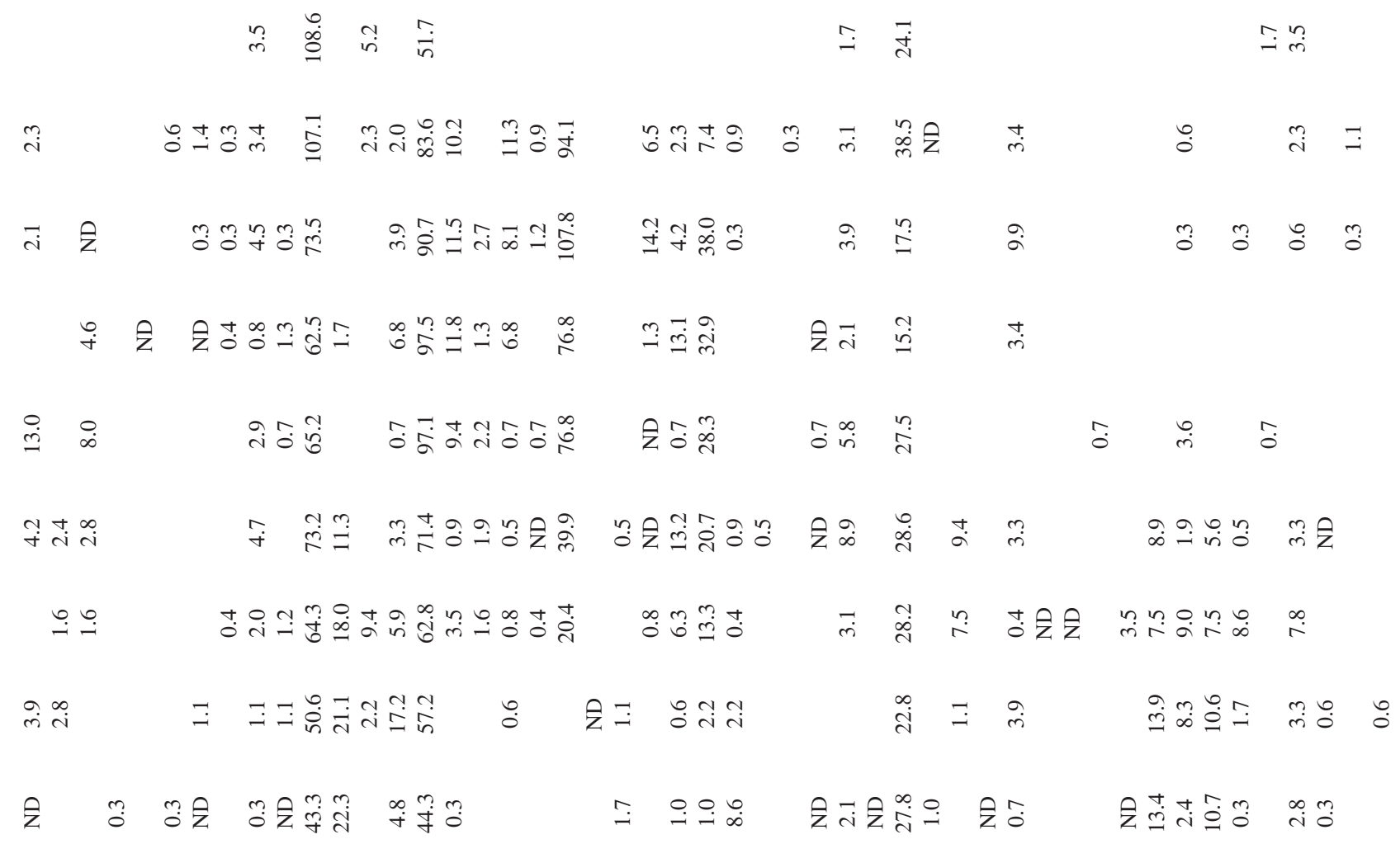

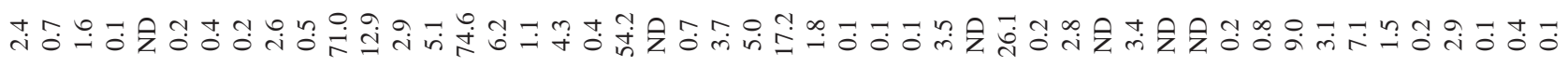

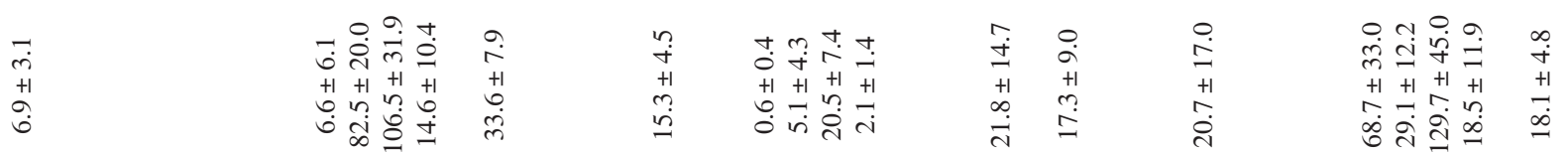

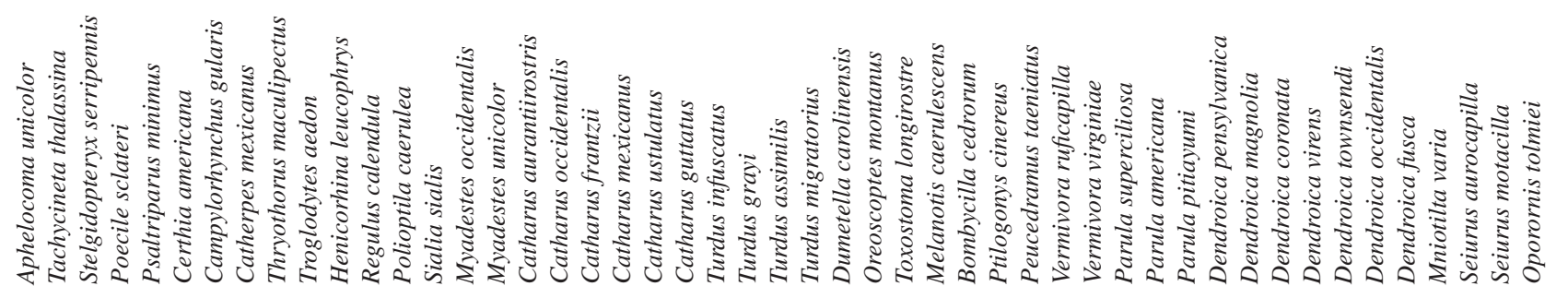




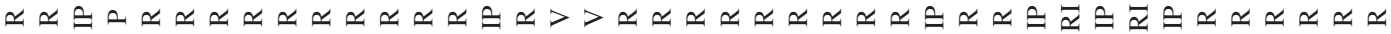

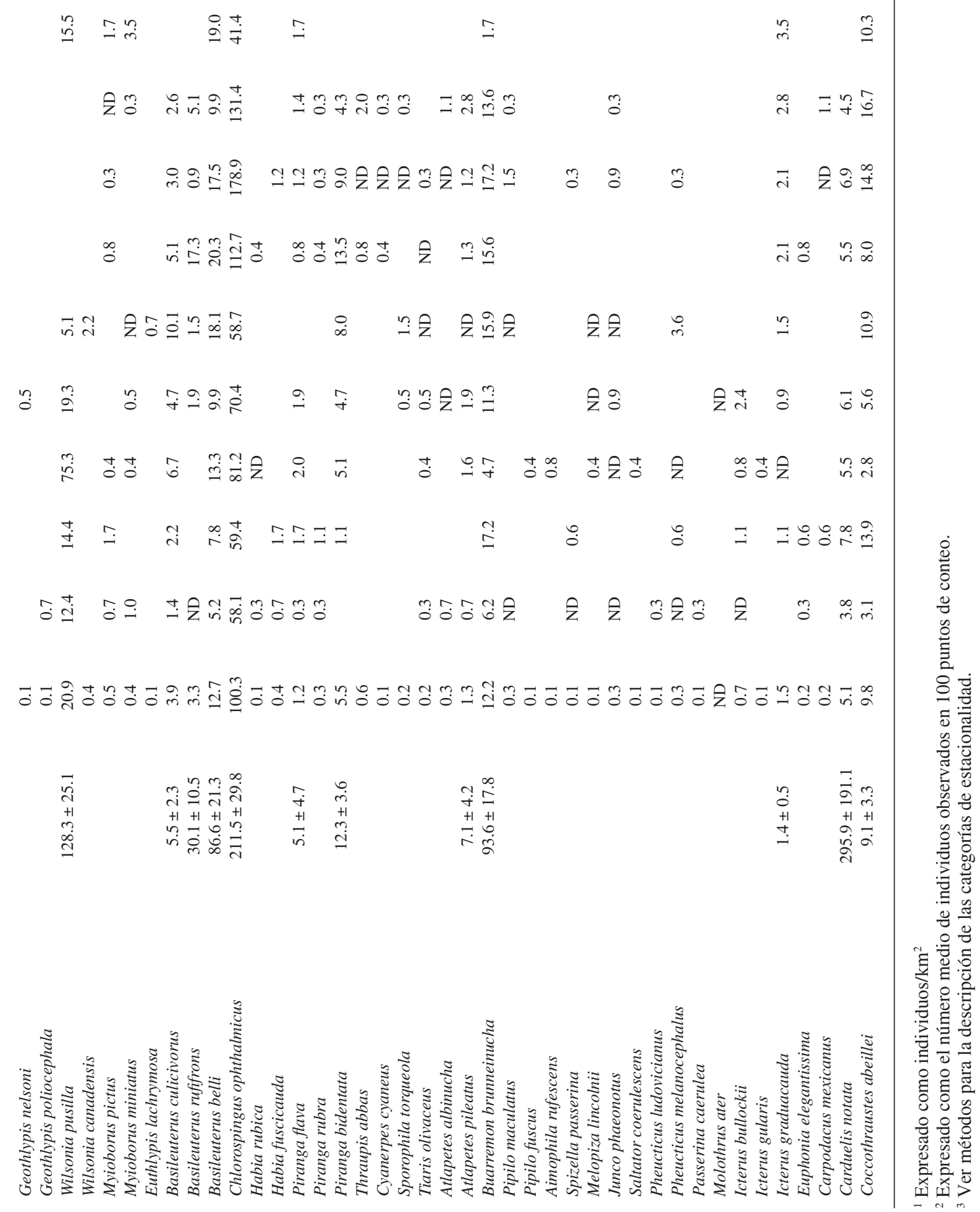




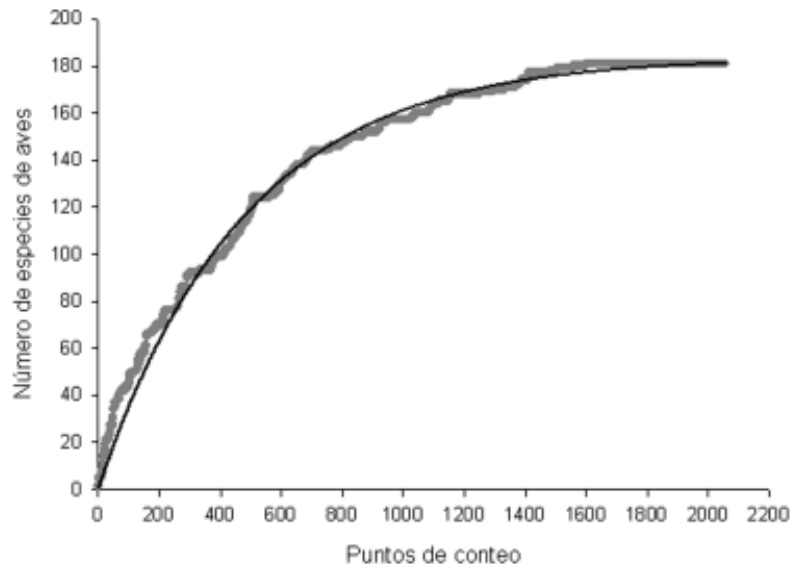

Figura 2. Curva de acumulación de especies de aves en función del esfuerzo de muestreo en el bosque mesófilo de montaña del noreste de Hidalgo, México. Los círculos grises representan los datos observados y la línea negra, la función exponencial de acumulación de especies.

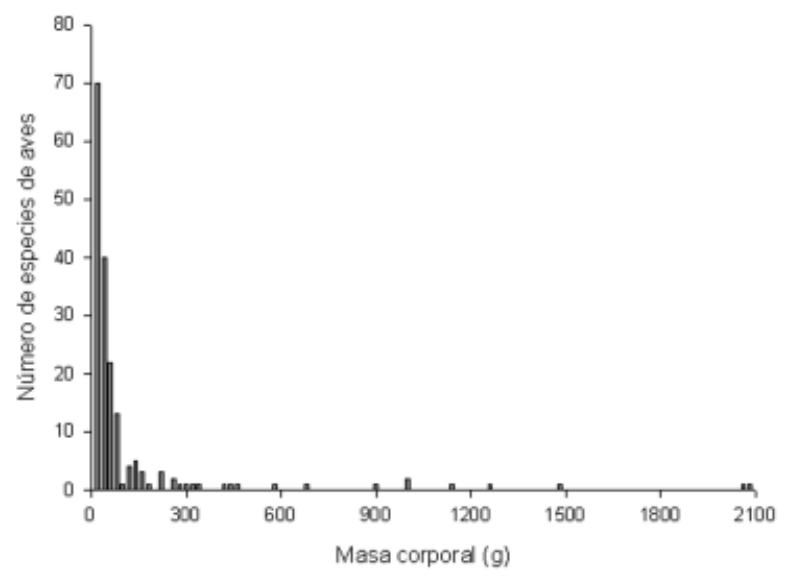

Figura 3. Distribución de la masa corporal en la comunidad de aves del bosque mesófilo de montaña del noreste de Hidalgo, México.

con 11 especies; de granívoros arborícolas con 11 especies de Emberizidae y Fringillidae, y de insectívoros-frugívoros arborícolas que atrapan al vuelo, con 11 especies de Momotidae y Tyrannidae. Asimismo, las rapaces diurnas, con 11 especies, constituyeron un gremio importante.

La mayor parte de las 181 especies registradas fueron residentes (132). Respecto a las migratorias, 5 fueron visitantes de verano, 7 con poblaciones residentes y visitantes de invierno, 29 con poblaciones visitantes de invierno y migratorias de paso y 6 , migratorias de paso (Cuadro 1).
De las 20 a 26 especies consideradas como restringidas o que usan preferentemente el bosque mesófilo en México (Howell y Webb, 1995; Escalante-Pliego et al., 1998), se detectaron 16 especies en el área de estudio: Dendrortyx barbatus, Geotrygon albifacies, Glaucidium sanchezi, Automolus rubiginosus, Sclerurus mexicanus, Xiphorhynchus erythropygius, Vireo leucophrys, Cyanolyca cucullata, Cyanolyca nana, Henicorhina leucophrys, Myadestes unicolor, Catharus mexicanus, Turdus infuscatus, Basileuterus belli, Chlorospingus ophthalmicus y Atlapetes albinucha. El área de distribución de las especies restantes no incluye el área de estudio, por lo que todas las especies consideradas como restringidas al bosque mesófilo en esta zona fueron detectadas.

En estos bosques mesófilos se detectaron 11 especies endémicas de México (Howell y Webb, 1995; Ceballos y Márquez-Valdelamar, 2000), y 3 de las 4 especies de distribución restringida del área de endemismo de aves Sur de la Sierra Madre Oriental (Stattersfield et al., 1998). Las endémicas de México fueron Dendrortyx barbatus, Aratinga holochlora, Glaucidium sanchezi, Atthis heloisa, Cyanolyca nana, Campylorhynchus gularis, Catharus occidentalis, Melanotis caerulescens, Geothlypis nelsoni, Atlapetes albinucha y Atlapetes pileatus. Las de distribución restringida, Dendrortyx barbatus, Glaucidium sanchezi y Cyanolyca nana.

Se detectaron 28 especies dentro de alguna categoría de riesgo de conservación, con base en la legislación mexicana vigente (SEMARNAT, 2002). Las catalogadas en peligro de extinción fueron Dendrortyx barbatus, Spizaetus ornatus, Glaucidium sanchezi y Cyanolyca nana. Las amenazadas, Penelope purpurascens, Geotrygon albifacies, Aratinga holochlora, Pionus senilis, Automolus rubiginosus, Xiphorhynchus erythropygius, Cyanolyca cucullata, Aphelocoma unicolor, Myadestes unicolor, Catharus frantzii, Turdus infuscatus y Oporornis tolmiei. Las consideradas bajo protección especial, Dactylortyx thoracicus, Chondrohierax uncinatus, Harpagus bidentatus, Buteogallus anthracinus, Buteogallus urubitinga, Micrastur semitorquatus, Trogon collaris, Aulacorhynchus prasinus, Campephilus guatemalensis, Sclerurus mexicanus, Myadestes occidentalis y Catharus mexicanus.

La abundancia relativa fue calculada para todas las especies detectadas en este estudio, excepto para 15 (Cuadro 1) que no fueron detectadas en los puntos de conteo, sino durante el desplazamiento entre los puntos. Las especies con los valores más altos de abundancia relativa, en orden de importancia, fueron Chlorospingus ophthalmicus, Myadestes occidentalis, Henicorhina leucophrys, Catharus mexicanus, Trogon mexicanus, Ptilogonys cinereus, Lepidocolaptes affinis, Melanerpes 
Cuadro 2. Número de especies y abundancia relativa de los gremios trófico conductuales de la comunidad de aves del bosque mesófilo de montaña del noreste de Hidalgo, México.

\begin{tabular}{|c|c|c|}
\hline Gremio trófico-conductual & Número de especies & Abundancia relativa $^{1}$ \\
\hline folívoro-frugívoro arborícola & 2 & 0.92 \\
\hline nectarívoro-insectívoro & 11 & 5.59 \\
\hline frugívoro arborícola & 6 & 2.58 \\
\hline frugívoro-nectarívoro arborícola & 1 & 0.10 \\
\hline frugívoro-insectívoro arborícola & 9 & 172.87 \\
\hline granívoro de suelo & 2 & 12.20 \\
\hline granívoro de sotobosque & 2 & 1.36 \\
\hline granívoro arborícola & 10 & 16.53 \\
\hline granívoro-frugívoro de suelo & 5 & 19.01 \\
\hline granívoro-frugívoro de sotobosque & 1 & 0.05 \\
\hline granívoro-frugívoro arborícola & 6 & 20.52 \\
\hline granívoro-frugívoro de borde de bosque & 1 & 0.05 \\
\hline granívoro-insectívoro arborícola & 1 & $<0.01$ \\
\hline insectívoro de suelo & 3 & 0.34 \\
\hline insectívoro de follaje de sotobosque & 3 & 73.65 \\
\hline insectívoro arborícola de follaje & 26 & 66.46 \\
\hline insectívoro arborícola que atrapa al vuelo & 7 & 39.96 \\
\hline insectívoro de superficie de corteza & 6 & 47.16 \\
\hline insectívoro de pared rocosa & 1 & 0.19 \\
\hline insectívoro aéreo diurno & 6 & 15.70 \\
\hline insectívoro aéreo nocturno & 2 & 0.58 \\
\hline insectívoro-nectarívoro arborícola de follaje & 5 & 5.40 \\
\hline insectívoro-frugívoro de suelo & 1 & 0.39 \\
\hline insectívoro-frugívoro de follaje de sotobosque & 6 & 58.87 \\
\hline insectívoro-frugívoro arborícola de follaje & 15 & 146.62 \\
\hline insectívoro-frugívoro arborícola que atrapa al vuelo & 10 & 18.28 \\
\hline insectívoro-frugívoro de interior de corteza & 7 & 27.95 \\
\hline consumidor de savia & 1 & 0.73 \\
\hline rapaz diurna & 10 & 3.01 \\
\hline rapaz nocturna & 1 & 0.49 \\
\hline piscívoro & 1 & $<0.01$ \\
\hline carroñero & 3 & 0.53 \\
\hline omnívoro de suelo & 1 & 1.12 \\
\hline omnívoro arborícola & 9 & 15.75 \\
\hline
\end{tabular}

${ }^{1}$ Expresado como el número medio de individuos observados en 100 puntos de conteo.

formicivorus, Wilsonia pusilla y Vireo leucophrys. La abundancia relativa de estas especies estuvo dentro de un intervalo de 20 a 100 individuos observados en 100 puntos de conteo. Estas 10 especies comprendieron el $58 \%$ del total de la abundancia relativa de esta comunidad de aves. La mayoría de las especies de aves fueron raras en términos de abundancia (detectadas en menos del $1 \%$ de los puntos de conteo), como se muestra en la figura 4.

Se obtuvieron estimaciones robustas de densidad sólo para 56 especies (Cuadro 1). Las especies con las densidades más altas, en orden de importancia, fueron Carduelis notata, Chlorospingus ophthalmicus, Dendroica townsendi, Wilsonia pusilla, Regulus calendula, Buarremon brunneinucha, Basileuterus belli, Henicorhina leucophrys, Eugenes fulgens y Dendroica coronata. La densidad de estas especies se calculó entre los 70 y 300 individuos/ $\mathrm{km}^{2}$ y pueden considerarse como las verdaderamente abundantes en el área de estudio, ya que sus estimaciones de densidad son independientes de su probabilidad de detección (Buckland et al., 1993, 2004). Excepto Buarremon brunneinucha, que tiene una masa corporal promedio de $47 \mathrm{~g}$, todas estas especies son pequeñas, ya que su masa corporal promedio oscila entre los 7 y $22 \mathrm{~g}$. Las especies que contribuyeron en mayor medida a la biomasa de esta comunidad fueron Chlorospingus ophthalmicus, Buarremon brunneinucha, Carduelis notata, Melanerpes formicivorus, Henicorhina leucophrys, Myadestes occidentalis, Trogon mexicanus, Turdus assimilis, Melanotis caerulescens y Patagioenas fasciata; las aves pequeñas, como Chlorospingus ophthalmicus, Carduelis notata y Henicorhina leucophrys, contribuyeron en gran medida debido a su gran abundancia, mientras que el resto 


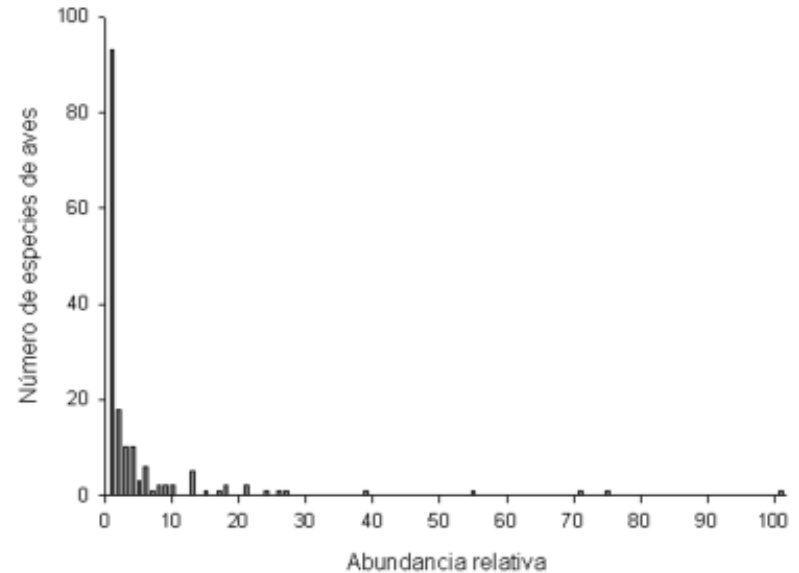

Figura 4. Distribución de la abundancia relativa en la comunidad de aves del bosque mesófilo de montaña del noreste de Hidalgo, México. La abundancia relativa está definida como el número promedio de individuos de una especie observados en 100 puntos de conteo.

de las especies contribuyeron de manera importante tanto por su abundancia, como por su masa corporal.

\section{Discusión}

El bosque mesófilo de montaña del noreste de Hidalgo mantiene una diversa comunidad de aves. Las 181 especies registradas representan el $40 \%$ de la avifauna estatal (Martínez-Morales, et al., 2007) lo cual es notable, en particular si se considera que el bosque mesófilo de montaña cubre aproximadamente el $7 \%$ de la superficie del estado (Velázquez et al., 2002). A pesar de los esfuerzos que a la fecha se han hecho por conocer la avifauna de este tipo de vegetación en Hidalgo, algunos de los sitios de bosque mesófilo que ya han sido inventariados, aún no se estudian de manera exhaustiva. Además, existen varios sitios importantes que prácticamente no han sido visitados, en especial los que se ubican en los municipios de Tlahuiltepa, San Bartolo Tutotepec y Tenango de Doria. La intensificación en el estudio de la comunidad de aves de este tipo de vegetación en el estado, indudablemente representaría un incremento en el registro de especies que utilizan este bosque, un mejor conocimiento de la distribución espacial y temporal de las especies, y mejores estimaciones de parámetros poblacionales, lo que redundaría en más información sobre la cual se podrían fundamentar estrategias de manejo para la conservación.

Las aves residentes fueron el grupo de especies más importante en esta comunidad de aves. A pesar de que el período de muestreo no incluyó algunos meses de la temporada de invernación (de octubre a diciembre), es probable que una gran proporción de las aves migratorias neotropicales hayan sido detectadas con base en la proporción registrada en otras localidades de bosque mesófilo en el este de México (Escalóna et al., 1995; Gram y Faaborg, 1997; Navarro et al., 2004). Por ejemplo, en este estudio el $20 \%$ de las especies fueron migratorias neotropicales, mientras que en la Reserva de la Biosfera El Cielo, Gram y Faaborg (1997) anotaron una proporción del 22\%; todas las especies que registran para El Cielo, fueron detectadas en el bosque mesófilo del noreste de Hidalgo, excepto Dendroica dominica y Hylocichla mustelina; aunque ésta última sólo fue capturada en redes de niebla en El Cielo. Esto podría sugerir que, a pesar de que en este estudio no fue cubierto por completo el período de invernación, la mayor parte de las especies fueron detectadas. Sin embargo, para aquellas especies migratorias que tienen su pico de abundancia entre octubre y diciembre, su importancia en términos de abundancia y biomasa podría estar subestimada, lo que podría estar reflejado en las bajas estimaciones de abundancia para la mayoría de la especies migratorias neotropicales que se registran en este estudio, en comparación con las estimaciones para las mismas especies en El Cielo.

Considerando la historia del impacto antrópico que ha ocurrido sobre el bosque mesófilo de esta región, no sería improbable que, a la fecha, algunas especies de aves hayan sufrido extinciones locales (Kattan et al., 1994). Por lo tanto, la comunidad de aves estudiada, no necesariamente constituiría la avifauna original, sino una comunidad ya depauperada en alguna medida. Es difícil evaluar esto de manera directa, ya que no existen inventarios históricos; sin embargo, se podrían hacer estimaciones a partir de la modelación de distribuciones potenciales de especies.

La biomasa en esta comunidad de aves demuestra una preponderancia de especies pequeñas. Es probable que esta preponderancia refleje una afectación sistemática en la estructura y composición de la vegetación del bosque que reduce el hábitat disponible para especies de mayor talla; por ejemplo, un dosel bajo y/o poco desarrollado, una simplificada estructura vertical de la vegetación, especies arbóreas de tallas reducidas, etc. (Thiollay, 1995). También podría responder a una productividad primaria relativamente baja, lo cual se traduciría en una baja biomasa y poca cantidad de individuos en niveles tróficos superiores (Robinson et al., 2000). Otro factor podría ser la cacería sesgada a especies de talla grande (e.g. Silva y Strahl, 1991, 1997; Redford, 1992; Jorgenson, 1995), lo que sugeriría que la comunidad de aves está relativamente depauperada. Las especies más raras incluyeron aquellas de talla grande o de talla grande para su gremio, tal es el caso de los crácidos, zopilotes, 7 de las rapaces diurnas y 
las especies Dendrortyx barabtus, Patagioenas flavirostris, Geotrygon montana, Pionus senilis, Ciccaba virgata, Momotus momota, Aulacorhynchus prasinus, Colaptes auratus, Campephilus guatemalensis y Xiphocolaptes promeropirhynchus. Es probable que algunas especies raras sean más comunes en otros tipos de vegetación que rodean al bosque mesófilo, como el bosque de pinoencino, las selvas tropicales, los acahuales (vegetación secundaria), pastizales y cultivos. También fueron raras las visitantes de verano y las migratorias de paso. Es importante señalar que algunas especies restringidas al bosque mesófilo fueron raras, tal es el caso de Dendrortyx barbatus, Geotrygon albifacies, Glaucidium sanchezi, Sclerurus mexicanus, Xiphorhynchus erythropygius, Cyanolyca nana y Atlapetes albinucha. Esto podría estar evidenciando un estado de conservación delicado para estas especies, lo que en términos de conservación es particularmente grave, pues la condición de rareza de estas especies, su preferencia por el bosque mesófilo, y el hecho de que este tipo de bosque sea un ecosistema muy restringido y fragmentado, podrían estar representando un preámbulo de extinción. Asimismo, dentro de estas especies están incluidas las 3 de distribución restringida, cuya extinción local exacerbaría su situación de conservación global. Esto enfatiza la urgente necesidad de llevar a cabo estrategias para la conservación del bosque mesófilo de montaña, al menos para Hidalgo, pues en el estado no se realizan acciones para el manejo de este frágil ecosistema. Por ejemplo, no existen áreas naturales protegidas que incluyan a este tipo de bosque, siendo que en el estado existen algunos de los remanentes más extensos de bosque mesófilo de la Sierra Madre Oriental. Si bien es cierto que la cobertura original del bosque mesófilo compite con otras formas de uso de suelo, éstas deben ser adecuadas con base en las características bióticas y abióticas de la zona. Existen numerosos trabajos que demuestran que en alguna medida los cultivos de café de sombra pueden funcionar como hábitat para numerosas especies de aves (Greenberg et al., 1997; Carlo et al., 2004; Tejeda-Cruz y Sutherland, 2004; Cruz-Angón y Greenberg, 2005), a diferencia de lo que ocurre actualmente en la región, en donde el bosque mesófilo es removido completamente para el establecimiento de pastizales, con el fin de introducir ganado vacuno.

\section{Agradecimientos}

A la gente de las comunidades que visité durante el trabajo de campo en Hidalgo, por su valiosa logística y amistad. Este manuscrito recibió atinadas observaciones de R. Ortiz-Pulido, C. Moreno, I. Zuria y de dos revisores anónimos. El estudio recibió el apoyo del Consejo Nacional de Ciencia y Tecnología (CONACYT), la Comisión Nacional para el Conocimiento y Uso de la Biodiversidad (CONABIO), British Airways a través del BA Assisting Conservation Programme, la Cambridge Philosophical Society y la Universidad Autónoma del Estado de Hidalgo.

\section{Literatura citada}

AOU (American Ornithologists' Union). 1998. Checklist of North American birds, séptima edición. American Ornithologists' Union, Washington, D.C.

AOU. 2000. Forty-second supplement to the American Ornithologists' Union check-list of North American birds. The Auk 117:847-858.

Arizmendi, M. del C. y L. Márquez-Valdelamar (eds.). 2000. Áreas de importancia para la conservación de las aves en México. Sección Mexicana del consejo Internacional para la preservación de las Aves, A. C. (CIPAMEX)/ Comisión Nacional para el Conocimiento y Uso de la Biodiversidad (CONABIO)/ Fondo Mexicano para la Conservación de la Naturaleza (FMCN), México, D.F. 157 p.

Arriaga, L., J. M. Espinoza, C. Aguilar, E. Martínez, L. Gómez y E. Loa (coordinadores). 2000. Regiones terrestres prioritarias de México. Comisión Nacional para el Conocimiento y Uso de la Biodiversidad, México, D.F.

Banks, R. C., C. Cicero, J. L. Dunn, A. W. Kratter, P. C. Rasmussen, J. V. Remsen Jr., J. D. Rising y D. F. Stotz. 2002. Forty-third supplement to the American Ornithologists' Union check-list of North American birds. The Auk 119:897-906.

Banks, R. C., C. Cicero, J. L. Dunn, A. W. Kratter, P. C. Rasmussen, J. V. Remsen Jr., J. D. Rising y D. F. Stotz. 2003. Forty-fourth supplement to the American Ornithologists' Union check-list of North American birds. The Auk 120:923-932.

Banks, R. C., C. Cicero, J. L. Dunn, A. W. Kratter, P. C. Rasmussen, J. V. Remsen Jr., J. D. Rising y D. F. Stotz. 2004. Forty-fifth supplement to the American Ornithologists' Union check-list of North American birds. The Auk 121:985-995.

Banks, R. C., C. Cicero, J. L. Dunn, A. W. Kratter, P. C. Rasmussen, J. V. Remsen Jr., J. D. Rising y D. F. Stotz. 2005. Forty-sixth supplement to the American Ornithologists' Union check-list of North American birds. The Auk 122:1026-1031.

Bates, D. M. y D. G. Watts. 1988. Nonlinear regression analysis and its applications. Wiley series in probability 
and mathematical statistics. Wiley, New York. 392 p.

Bjelland, A. D. y J. C. Ray. 1977. Birds collected in the state of Hidalgo, Mexico. Occasional Papers of the Museum of Texas Tech University 46:1-32.

Buckland, S. T., D. R. Anderson, K. P. Burnham y J. L. Laake. 1993. Distance sampling: estimating abundance of biological populations. Chapman and Hall, London. $446 \mathrm{p}$.

Buckland, S. T., D. R. Anderson, K. P. Burnham, J. L. Laake, D. L. Borchers y L. Thomas. 2004. Advanced distance sampling. Estimating abundance of biological populations. Oxford University. 416 p.

Carlo, T. A., J. A. Collazo y M. J. Groom. 2004. Influences of fruit diversity and abundance on bird use of two shaded coffee plantations. Biotropica 36:602-614

Ceballos G. y L. Márquez-Valdelamar. 2000. Las aves de México en peligro de extinción. UNAM/CONABIO/ Fondo de Cultura Económica, México, D.F. 430 p.

Cruz-Angón, A., y R. Greenberg. 2005. Are epiphytes important for birds in coffee plantations? An experimental assessment. Journal of Applied Ecology 42:150-159.

Challenger, A. 1998. Utilización y conservación de los ecosistemas terrestres de México. Pasado, presente y futuro. CONABIO/ Instituto de Biología,UNAM, Agrupación Sierra Madre, México, D.F., p. 446-457.

Chapman, F. M. 1898. Notes on birds observed at Jalapa and Las Vigas, Vera Cruz [Veracruz], Mexico. Bulletin of the Museum of Natural History 10:15-43.

Dunning, B. J. Jr. 1993. CRC Handbook of avian body masses. New York. 384 p.

Escalante-Pliego, P., A. G. Navarro-Sigüenza y A. T. Peterson. 1998. Un análisis geográfico, ecológico e histórico de la diversidad de aves terrestres de México. In Diversidad biológica de México: orígenes y distribución, T. P.Ramammoorthy, R. Bye, A. Lot, y J. Fa (eds.). Instituto de Biología, UNAM, México, D.F. p. 279-304.

Escalona, G., M. Torres, A. G. Navarro, R. Villalon, B. Hernández y H. Benítez. 1995. Migratory birds of the cloud forest of México. In Conservation of Neotropical migratory birds in Mexico, M. H. Wilson y S. A. Sader (eds.). Maine Agricultural and Forest Experiment Station, Miscellaneous Publication 727. p. 15-33.

Gram, W. K. y J. Faaborg. 1997. The distribution of Neotropical migrant birds wintering in the El Cielo Biosphere Reserve, Tamaulipas, Mexico. The Condor 99:658-670.

Greenberg, R., P. Bichier y J. Sterling. 1997. Bird populations in rustic and planted shade coffee plantations of eastern Chiapas, Mexico. Biotropica 29:501-514.
Hernández-Baños, B. E., A. T. Peterson, A. G. NavarroSigüenza y B. P. Escalante-Pliego. 1995. Bird faunas of the humid montane forests of Mesoamerica: biogeographic patterns and priorities for conservation. Bird Conservation International 5:251-277.

Howell, S. N. G. y S. Webb. 1992. A little-known cloud forest in Hidalgo, Mexico. Euphonia 1:7-11

Howell, S. N. G. y S. Webb. 1995. A guide to the birds of Mexico and northern Central America. Oxford University Press, Nueva York. 851 p.

Jorgenson, J. P. 1995. Maya subsistence hunters in Quintana Roo, Mexico. Oryx 29:49-57.

Kattan, G. H., H. Alvarez-López y M. Giraldo. 1994. Forest fragmentation and bird extinctions: San Antonio eighty years later. Conservation Biology 8:138-146.

Lowery, G. H., Jr. y R. J. Newman. 1949. New birds from the state of San Luis Potosi and the Tuxtla Mountains of Veracruz, Mexico. Louisiana State University Museum, Occasional Papers 22:1-10.

Luna-Vega, I., S. Ocegueda-Cruz y O. Alcántara-Ayala. 1994. Florística y notas biogeográficas del bosque mesófilo de montaña del municipio de Tlanchinol, Hidalgo, México. Anales del Instituto de Biología, Universidad Nacional Autónoma de México, Serie Botánica 65:31-62.

Luna-Vega, I., O. Alcántara-Ayala, J. J. Morrone y D. Espinosa-Organista. 2000. Track analysis and conservation priorities in the cloud forest of Hidalgo, Mexico. Diversity and Distributions 6:137-143.

Martin, P. S. 1955. Zonal distribution of vertebrates in a Mexican cloud forest. The American Naturalist 89:347-361.

Martínez-Morales, M. A. 2001. Forest fragmentation effects on bird communities of tropical montane cloud forests in eastern Mexico. Tesis de Doctorado, University of Cambridge. 229 p.

Martínez-Morales, M. A. 2004. Nuevos registros de aves en el bosque mesófilo de montaña del noreste de Hidalgo, México. Huitzil 5:12-19.

Martínez-Morales, M. A. 2005a. Landscape patterns influencing bird assemblages in a fragmented Neotropical cloud forest. Biological Conservation 121:117-126.

Martínez-Morales, M. A. 2005b. Nested species assemblages as a tool to detect sensitivity to forest fragmentation: the case of cloud forest birds. Oikos 108: 634-642.

Martínez-Morales, M. A., R. Ortiz-Pulido, B. de la Barreda, I. Zuria, J. Bravo-Cadena y J. ValenciaHerverth. 2007. HIDALGO. In Avifaunas estatales de México, R. Ortiz-Pulido, A. Navarro-Sigüenza, H. Gómez de Silva, O. Rojas-Soto y T. A. Peterson (eds.). 
CIPAMEX. Pachuca, p. 49-95.

Miranda, F. y A. J. Sharp. 1950. Characteristics of the vegetation in certain temperate regions of eastern Mexico. Ecology 31:313-333.

Navarro, A. G. 1992. Altitudinal distribution of birds in the Sierra Madre del Sur, Guerrero, Mexico. The Condor 94:29-39.

Navarro, A. G., J. E. Morales-Pérez y B. E. Hernández Baños. 1991. Aves de Teocelo. Teocelo 7/8:11-34.

Navarro, A. G., H. A. Garza-Torres, S. López de Aquino, O. R. Rojas-Soto y L. A. Sánchez-González. 2004. Patrones biogeográficos de la avifauna.In Biodiversidad de la Sierra Madre Oriental, I. Luna, J. J. Morrone y D. Espinosa (eds.). CONABIO/UNAM, México, D.F. p. 439-467.

Nocedal, J. 1994. Local migrations of insectivorous birds in western Mexico: implications for the protection and conservation of their habitats. Bird Conservation International 4:129-142.

Ralph, C. J., J. R. Saber y S. Droege. (editores técnicos). 1995. Monitoring bird populations by point counts. Gen. Tech. Rep. PSW-GTR-149. Albany, CA: Pacific Southwest Research Station, Forest Service, U.S. Department of Agriculture. $181 \mathrm{p}$.

Redford, K. H. 1992. The empty forest. BioScience 42:412-422.

Robinson, W. D., J. D. Brawn y S. K. Robinson. 2000. Forest bird community structure in central Panama: influence of spatial scale and biogeography. Ecological Monographs 70:209-235.

Rojas-Soto, O., L. A. Sánchez-González y S. López de Aquino. 2002. New information on birds of northern Hidalgo, Mexico. Southwestern Naturalist 47:471475.

Rzedowski, J. 1981. Vegetación de México. Limusa, México, D.F., p. 315-326.

SEMARNAT (Secretaría de Medio Ambiente y Recursos Naturales) 2002. Norma Oficial Mexicana NOM-059ECOL-2001, Protección ambiental - Especies nativas de México de flora y fauna silvestres - Categorías de riesgo y especificaciones para su inclusión, exclusión o cambio - Lista de especies en riesgo. Diario Oficial de la Federación, segunda sección; 6 de marzo. p. $1-81$.

Silva, J. L. y S. D. Strahl. 1991. Human impact on populations of chachalacas, guans and curassows (Galliformes: Cracidae) in Venezuela. In Neotropical wildlife use and conservation, J. G. Robinson and K. H. Redford. (eds.). The University of Chicago, Chicago. p. 37-52.

Silva, J. L. y S. D. Strahl. 1997. Presión de caza sobre poblaciones de crácidos en los parques nacionales al norte de Venezuela. In The cracidae: their biology and conservation, S. D. Strahl, S. Beaujon, D. M. Brooks, A. J. Begazo, G. Sedaghatkish, y F. Olmos. (eds.). Hancock House, Washington. p. 437-438.

Soberón, J. y J. Llorente. 1993. The use of species accumulation functions for the prediction of species richness. Conservation Biology 7:480-488.

Stattersfield, A. J., M. J. Crosby, A. J. Long y D. C. Wege. 1998. Endemic bird areas of the world. Priorities for biodiversity conservation. BirdLife Conservation Series No. 7. Birdlife International, Cambridge, p. 120-121.

Sutton, G. M. y T. D. Burleigh. 1940. Birds of Valles, San Luis Potosi, Mexico. The Condor 2:259-262.

Tejeda-Cruz, C. y W. J. Sutherland. 2004. Bird responses to shade coffee production. Animal Conservation 7:169-179.

Terborgh, J., S. K Robinson, T. A. Parker III, C. A. Munn y N. Pierpont. 1990. Structure and organization of an Amazonian forest bird community. Ecological Monographs 60:213-238.

Thomas, L., J. L. Laake, S. Strindberg, F. F. C. Marques, S. T. Buckland, D. L. Borchers, D. R. Anderson, K. P. Burnham, S. L. Hedley, J. H. Pollard, J. R. B. Bishop and T. A. Marques. 2005. Distance 5.0. Beta 4. Research Unit for Wildlife Population Assessment, University of St. Andrews, St. Andrews. Disponible en: http://www.ruwpa.st-and.ac.uk/distance/

Thiollay, J. M. 1995. The role of traditional agroforests in the conservation of rain forest bird diversity in Sumatra. Conservation Biology 9:335-353.

Velázquez, A., J. F. Mas y J. L. Palacio. 2002. Análisis del cambio de uso del suelo. Instituto de Geografía,UNAM,/ Instituto Nacional de Ecología, SEMARNAT, México, D.F.

Wetmore, A. 1943. The birds of southern Veracruz, Mexico. Proceedings of the United States National Museum 93:215-340.

Williams-Linera, G., A. Rowden y A. C. Newton. 2003. Distribution and stand characteristics of relict populations of Mexican beech (Fagus grandifolia var. mexicana). Biological Conservation 109:27-36. 